\title{
Risk Factors for Peri-Implantitis: Effect of History of Periodontal Disease and Smoking Habits. A Systematic Review and Meta-Analysis
}

\author{
Claudio Stacchi ${ }^{1}$, Federico Berton ${ }^{1}$, Giuseppe Perinetti ${ }^{1}$, Andrea Frassetto ${ }^{1}$, Teresa Lombardi ${ }^{2}$, \\ Aiman Khoury ${ }^{1}$, Francesca Andolsek ${ }^{3}$, Roberto Di Lenarda ${ }^{1}$
}

${ }^{1}$ Department of Medical, Surgical and Health Sciences, University of Trieste, Italy.

${ }^{2}$ Private Practice, Cassano allo Ionio, Italy.

${ }^{3}$ Private Practice, Trieste, Italy.

\author{
Corresponding Author: \\ Claudio Stacchi \\ Clinica Odontoiatrica e Stomatologica dell’Università degli Studi di Trieste \\ Piazza Ospitale, 1 - 34100 Trieste \\ Italy \\ Phone: +39-0481-531229 \\ E-mail: claudio@stacchi.it
}

\begin{abstract}
Objectives: The purpose of this review was to evaluate whether history of periodontitis and smoking habits could represent a risk factor for peri-implantitis and implant loss.

Material and Methods: This systematic review followed PRISMA guidelines and was registered at the PROSPERO database [registration numbers CRD42016034160 (effect of history of periodontitis) and CRD42016033676 (effect of smoking)]. Broad electronic (MEDLINE) and manual searches were conducted among articles published from January $1^{\text {st }} 1990$ up to December $31^{\text {st }} 2015$, resulting in 49332 records for history of periodontitis and 3199 for smoking habits. Selection criteria included prospective studies comparing two cohorts of patients, with and without the investigated risk factor, with a minimum followup period of three years, and reporting data on peri-implantitis and implant loss occurrence. Considering that only prospective studies were included, dichotomous data were expressed as risk ratios and $95 \%$ confidence intervals.

Results: Three studies evaluating history of periodontitis (on which quantitative analysis was performed) and one study on smoking effect were included. Both implant and patient-based meta-analyses revealed a significantly higher risk of developing peri-implantitis in patients with a history of periodontitis compared with periodontally healthy subjects, but not a statistically significant increased risk for implant loss.

Conclusions: The outcomes of this systematic review indicate history of periodontitis as a possible risk factor for periimplantitis, while insufficient data are present in literature to evaluate the role of smoking. However, available evidence is still weak and immature, and sound epidemiological studies are needed to analyse the specific contribution of these potential risk factors.
\end{abstract}

Keywords: dental implants; peri-implantitis; periodontitis; risk factors; smoking; systematic review.

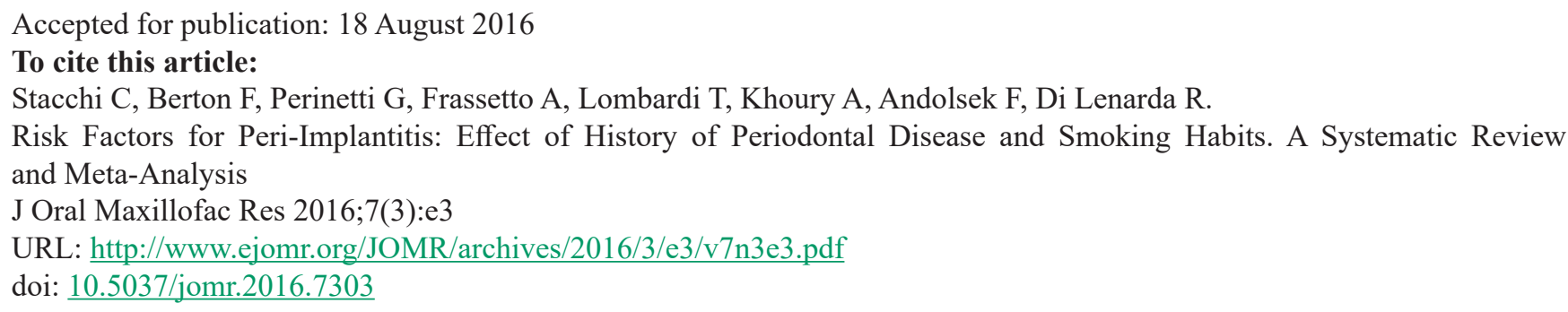




\section{INTRODUCTION}

Over the last decades, the use of implant-supported dental rehabilitations has known a significant increase [1]. Despite a high overall success rate, various risk factors can negatively affect the predictability of dental implants, leading to peri-implant tissue inflammation, bone resorption and, ultimately, to implant loss. Among them, history of periodontal disease and smoking habits have often been identified as conditions favouring the onset of peri-implant pathologies [2-3]. Even if several longitudinal studies have been conducted on these issues, showing some evidence of a negative impact on implant success [4-6], recent systematic reviews on this topic did not draw definitive conclusions [7-10].

The influence of the different risk factors, together with their specific weight and role in favouring periimplant disease, needs to be fully clarified to elucidate the health/disease process affecting the marginal tissues surrounding dental implants. Strict disease definitions, accurate stratification of the study groups and control of the confounders are crucial points to design appropriate trials, in order to evaluate the impact of each single risk factor in promoting the development of this multifactorial pathology.

The aim of this systematic review was to assess current scientific evidence regarding history of periodontitis and smoking habits as risk factors for implant loss and incidence of peri-implantitis, applying stringent selection criteria for study inclusion and quality assessment.

\section{MATERIAL AND METHODS \\ Protocol and search strategy}

The present systematic review followed the Preferred Reporting Items for Systematic Reviews and MetaAnalyses (PRISMA) statement [11], and it has been registered at the PROSPERO database (www.crd. york.ac.uk/PROSPERO) with registration numbers CRD42016034160 (effect of history of periodontitis) and CRD42016033676 (effect of smoking).

\section{Focus questions}

The following PICO (Patient, Intervention, Comparison and Outcome) questions were aimed to be answered:

1. In patients with osseointegrated dental implants, does a history of periodontal disease influence the occurrence of peri-implantitis and implant loss compared to a history of periodontal health?

Population: patients with osseointegrated dental implants.

Intervention or exposure: patients with a history of periodontal disease.

Comparison: periodontally healthy patients.

Outcome: occurrence of peri-implantitis/implant loss.

2. In patients with osseointegrated dental implants, does the presence of smoking habits influence the occurrence of peri-implantitis and implant loss compared to no smokers?

Population: patients with osseointegrated dental implants.

Intervention or exposure: smoker patients.

Comparison: no smoker patients.

Outcome: occurrence of peri-implantitis/implant loss.

\section{Information sources}

A broad electronic search was conducted on MEDLINE (PubMed, www.ncbi.nlm.nih.gov/ pubmed) by two independent authors (AF and TL), selecting articles published from January $1^{\text {st }} 1990$ up to the latest access on December $31^{\text {st }} 2015$. A manual search among the references of all full text articles and reviews emerging from the electronic search was also performed. No language restriction was applied, in order to limit selection bias.

\section{Search}

Search in the selected electronic database was performed by using the following algorithms.

1. Effect of history of periodontitis:

$((()((()(($ “dental implants”) OR “dental implant”) OR "peri-implantitis") OR "peri-implant bone") OR "perimplantitis") AND "periodontitis") OR “chronic periodontitis") OR "aggressive periodontitis") OR "periodontal disease") OR "periodontal pocket") OR "attachment loss") OR "alveolar bone loss").

2. Effect of smoking:

((()((()(("peri-implantitis") OR “perimplantitis”) OR "peri-implant disease") OR "peri-implant inflammat*") OR "perimplant inflammat*") OR "periimplant mucositis") OR "perimplant mucositis") OR "dental implant disease") OR "dental implant inflammation") OR "oral implant disease") OR "oral implant inflammation").

\section{Selection of studies}

Two blinded authors (CS and FB) independently performed the eligibility assessment of the studies. Intra-examiner reliability in the study selection 
process was assessed through the Cohen k-test assuming a threshold value of 0.61 [12]. Conflicts were resolved by discussion of each article, until consensus was reached. An attempt to contact the corresponding Authors of the included studies was made, in order to retrieve any missing information or to clarify specific items.

\section{Types of publications}

This systematic review included prospective studies conducted on human subjects. Systematic reviews and studies of lower quality within the hierarchy of scientific evidence (such as $\mathrm{PhD}$ theses, expert opinions, letters, editorials, case reports, case series, and retrospective studies) were excluded.

\section{Types of studies}

Only prospective studies with two cohorts of patients (patients with periodontal disease compared to periodontally healthy/smokers compared to no smokers) reporting data on the incidence of periimplantitis and implant loss in the two groups and complying with inclusion criteria listed below were included.

\section{Types of participants/population}

Subjects analysed in the included studies must have had at least one osseointegrated titanium screwshaped dental implant followed at least for three years.

\section{Inclusion and exclusion criteria}

Full texts of the possibly relevant studies were evaluated for selection according to the following inclusion criteria:

- Prospective cohort studies comparing an exposed with a not exposed cohort (patients with periodontal disease compared to periodontally healthy/smokers compared to no smokers);

- Studies reporting the incidence of implant biological complications in the cohorts;

- Studies reporting the incidence of implant loss in the cohorts;

- Studies reporting a clear definition of periimplantitis;

- Studies reporting a clear definition of periodontal disease (for part a) Effect of history of periodontitis);

- Studies reporting a clear definition of smoking habits (for part b) Effect of history of smoking);

- Minimum follow-up period of three years;
- Minimum of 10 evaluated implants per cohort.

The following exclusion criteria were applied:

- Animal or in vitro studies;

- Studies involving patients with systemic conditions potentially influencing dental implants outcome (e.g. immunologic disorders, uncontrolled diabetes mellitus, osteoporosis, HIV positive);

- Studies investigating dental implants manufactured in materials different from titanium;

- Articles published prior to January 1, 1990.

\section{Sequential search strategy}

Following the initial literature search, all article titles were screened to eliminate irrelevant publications, in vitro and animal studies, case reports, case series, retrospective studies and review articles. Then, studies were further selected basing on data obtained from the screening of the abstracts. In the final stage, the full texts of the selected papers were examined to confirm study eligibility, following inclusion and exclusion criteria.

\section{Data extraction}

Data were independently extracted from selected studies in form of variables by two authors (CS and FB), according to the aims and themes of the present review, as listed below.

\section{Data items}

The following items were extracted by using predefined forms: i) year of publication, ii) study design, iii) sample size, iv) gender distribution, v) mean age or age range, vi) number of implants, vii) smoking habits, viii) definition of peri-implantitis, ix) definition of periodontal disease, and $\mathrm{x}$ ) other confounding factors. Moreover, primary outcomes included: i) incidence of peri-implantitis: number of events between baseline and end of the follow-up period, evaluated on a patient and implant level, ii) implant loss: number of events between baseline and end of follow-up period in the cohorts, evaluated on a patient and implant level. The corresponding $95 \%$ confidence intervals (CIs) were also calculated including continuity correction.

\section{Assessment of risk of bias in individual studies}

Risk of bias in individual studies was assessed by two independent blinded examiners (FA and TL) according to the Newcastle-Ottawa scale (NOS) for cohort 
studies [13]. The NOS evaluates the study quality on the basis of three major components: selection, comparability, and outcome. It assigns up to four stars for selection, two stars for comparability, three stars for outcome. According to this analysis, a maximum of nine stars/points can be given to an observational study, with this score representing the highest quality, where six or more points are considered associated to a high quality study.

\section{Assessment of risk of bias across studies}

Heterogeneity was assessed using the $\chi^{2}$-based Q-statistic method with a significant $\mathrm{P}$ value $<0.1$. However, because of the moderate insensitivity of the Q statistic [14], an $\mathrm{I}^{2}$ index was also reported with values $\geq 50 \%$ considered associated to a substantial heterogeneity among the studies [15]. In particular, the $\mathrm{I}^{2}$ index describes the percentage of total variation across studies due to heterogeneity rather than chance. The $\tau^{2}$ was also calculated for the heterogeneity assessment. The Review Manager software (version 5.2.6 - http://www.cochrane.org) was used for the assessment of heterogeneity. Due to the limited number of included studies, additional investigations regarding publication bias or sensitivity analysis were not performed.

\section{Statistical analysis}

Risk ratios (RRs) were used for statistical pooling of data and results were expressed as mean and $95 \%$ CIs. Moreover, to account for any heterogeneity of the studies, a random effect model was used for calculations of all the overall effects [16]. Finally, these analyses were reported both for the implant loss incidence between exposed and not exposed patients, and for the peri-implantitis incidence between exposed and not exposed patients. In particular, the main outcomes were reported and analysed using both implant and patient as statistical unit. The Review Manager software (version 5.2.6 - www.cochrane.org) was used for meta-analysis.

\section{RESULTS \\ Search results \\ Effect of history of periodontitis}

A total of 58037 articles (in English, German, Chinese, Korean, Russian, Dutch, Italian and French languages) were found through electronic and manual searches (57960 in Medline and 77 manually) and, after removing duplicates, 49332 publications were evaluated (Cohen k-test for inter-reviewer agreement $=0.81$ ). After examination of titles and abstracts, 49312 articles were excluded and twenty papers were examined in full text to assess their eligibility (interreviewer agreement $=0.87$ ). Seventeen articles were excluded in the selection process [4-6, 17-30] and three studies [31-33] were included in this systematic review (inter-reviewer agreement $=1$ ). The first included study was published in 2003 [31], the last one in 2012 [33]. Median year of publication was 2008.

\section{Effect of smoking}

A total of 3719 articles (in English, German, Chinese, Russian, Italian and French languages) were found through electronic and manual searches (3696 in Medline and 23 manually) and, after removing duplicates, 3199 publications were evaluated (Cohen $\mathrm{k}$-test for inter-reviewer agreement $=0.88$ ). After examination of titles and abstracts, 3167 articles were excluded and thirty-two works were examined in full text to assess their eligibility (inter-reviewer agreement $=0.87$ ). Thirty-one articles were excluded in the selection process $[\underline{6}, \underline{27}, \underline{29}, \underline{30}, \underline{33-59}]$ and one study [31] was included in this systematic review (inter-reviewer agreement $=1$ ). The included study was published in 2003 [31].

The results of the electronic and manual searches are summarised in Figure 1. The list of the excluded studies and reasons for exclusion are provided in Table 1 and 2.

\section{Study characteristics \\ Effect of history of periodontitis}

The sample size in the single studies ranged from a minimum of $53[31,33]$ to a maximum of 62 [32] patients. The total number of treated patients was 168 (68 females, 47 males and 53 not specified). One study [31] did not report the sex distribution. Age range varied from 18 [32] to 85 [32] years old. Overall mean age was 49.7 years. One study [31] did not report the mean age of the patients. On the total of 168 patients, 92 subjects were periodontally healthy $(54.8 \%)$, while patients with a history of periodontitis were $76(45.2 \%)$. These 76 patients had different histories of periodontal disease: eight patients were affected by chronic periodontitis (10.5\%) [31], seven patients by moderate periodontitis $(9.2 \%)$ [32], 26 patients by severe periodontitis (34.2\%) [32] and 35 subjects by generalised aggressive periodontitis (46.1\%) [33]. The total number of inserted implants was 518 (193 in periodontally healthy patients, 325 in patients with a history of periodontitis). 


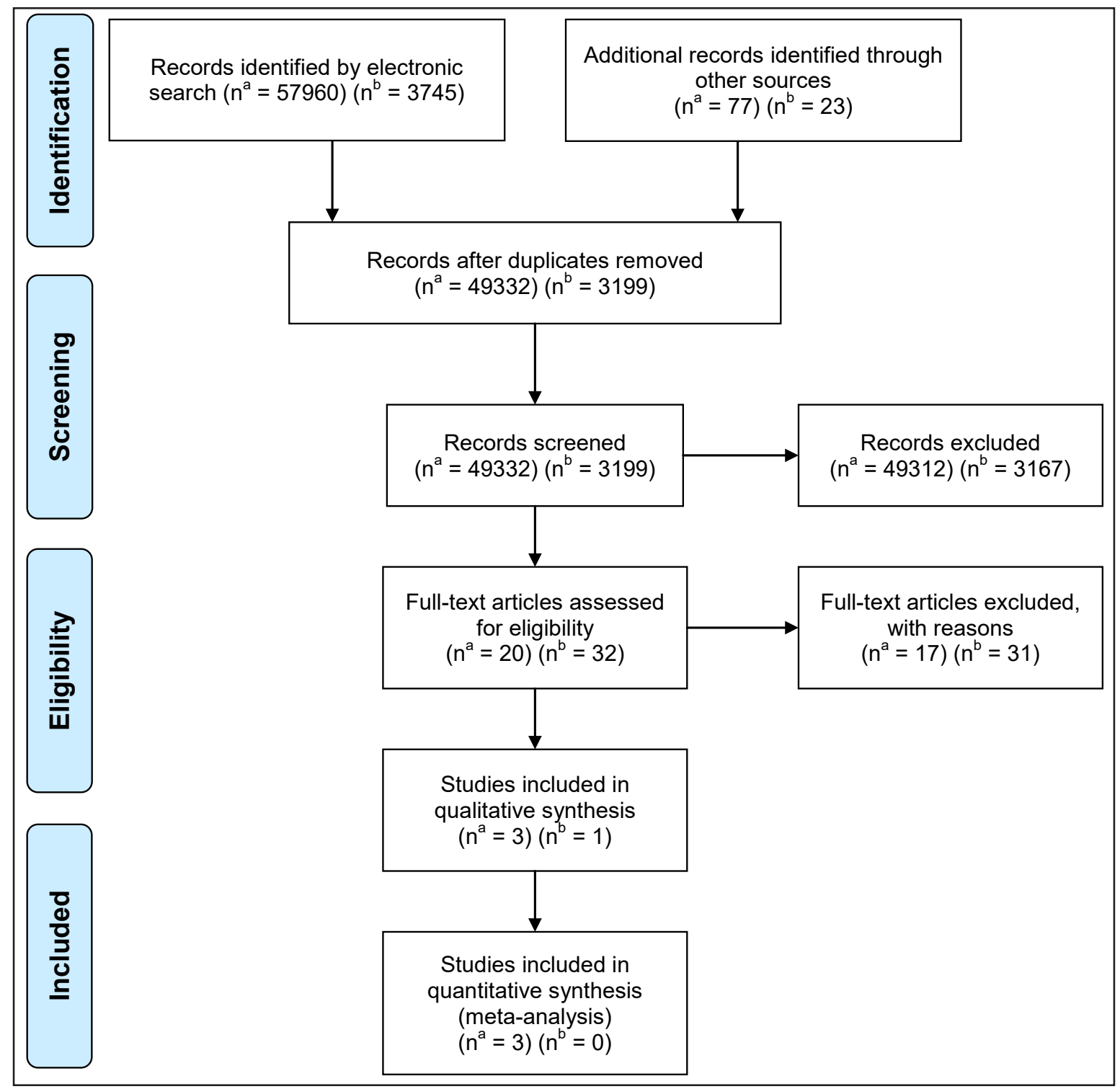

Figure 1. PRISMA flow diagram.

${ }^{a}$ number of records for history of periodontitis. ${ }^{b}$ number of records for smoking.

Table 1. List of excluded studies and reasons for exclusion (history of periodontitis)

\begin{tabular}{l|c|c|l}
\hline \multicolumn{1}{c|}{ Study } & $\begin{array}{c}\text { Year of } \\
\text { publication }\end{array}$ & Type of study & \multicolumn{1}{c}{ Reason of exclusion } \\
\hline Ferreira et al. [4] & 2006 & Cross sectional & Not prospective \\
\hline Roccuzzo et al. [5] & 2010 & Prospective & Did not report information on peri-implantitis \\
\hline Kostantinidis et al. [6] & 2015 & Cross sectional & Not prospective \\
\hline Sbordone et al. [17] & 1999 & Prospective & No control group \\
\hline Mengel et al. [18] & 2001 & Prospective & No control group \\
\hline Mengel et al. [19] & 2005 & Prospective & Did not report information on peri-implantitis \\
\hline Mengel et al. [20] & 2007 & Prospective & Did not report information on peri-implantitis \\
\hline Fardal and Linden [21] & 2007 & Prospective & No control group \\
\hline De Boever et al. [22] & 2009 & Prospective & Peri-implantitis criteria not clearly defined \\
\hline Koldsland et al. [23] & 2009 & Cross sectional & Not prospective \\
\hline Levin et al. [24] & 2011 & Prospective & Did not report information on peri-implantitis \\
\hline Horwitz and Machtei [25] & 2012 & Prospective & No control group \\
\hline Pjetursson et al. [26] & 2012 & Prospective & No control group \\
\hline Casado et al. [27] & 2013 & Retrospective & Not prospective \\
\hline Jiang et al. [28] & 2013 & Prospective & Did not report information on peri-implantitis \\
\hline Marrone et al. [29] & 2013 & Cross sectional & Not prospective \\
\hline Roccuzzo et al. [30] & 2014 & Prospective & Peri-implantitis criteria not clearly defined \\
\hline
\end{tabular}


Table 2. List of excluded studies and reasons for exclusion (smoking habits)

\begin{tabular}{|c|c|c|c|}
\hline Study & $\begin{array}{c}\text { Year of } \\
\text { publication }\end{array}$ & Type of study & Reason of exclusion \\
\hline Kostantinidis et al. [6] & 2015 & Cross sectional & Not prospective \\
\hline Casado et al. [27] & 2013 & Retrospective & Not prospective \\
\hline Marrone et al. [29] & 2013 & Cross-sectional & Not prospective \\
\hline Roccuzzo et al. [30] & 2014 & Prospective & Did not compare smokers vs. no smokers \\
\hline Swierkot et al. [33] & 2012 & Prospective & Did not compare smokers vs. no smokers \\
\hline Roos-Jansåker [34] & 2006 & Prospective & Did not compare smokers vs. no smokers \\
\hline Costa et al. [35] & 2012 & Follow up study & Did not report information on smoking \\
\hline Dvorak et al. [36] & 2011 & Cross sectional & Not prospective \\
\hline Schropp et al. [37] & 2014 & Randomized clinical trial & Did not compare smokers vs. no smokers \\
\hline Göthberg et al. [38] & 2014 & Randomized clinical trial & Did not report information on peri-implantitis \\
\hline Sanz et al. [39] & 2015 & Randomized clinical trial & Did not report information on smoking \\
\hline Cecchinato et al. [40] & 2014 & Prospective & Did not compare smokers vs. no smokers \\
\hline Wang et al. [41] & 2014 & Cross sectional & Not prospective \\
\hline Meijer et al. [42] & 2014 & Prospective & Did not report information on smoking \\
\hline Canullo et al. [43] & 2016 & Cross sectional & Not prospective \\
\hline Aguilar-Salvatierra et al. [44] & 2016 & Prospective & Did not report information on smoking \\
\hline Serino et al. [45] & 2015 & Prospective & Did not compare smokers vs. no smokers \\
\hline Mangano et al. [46] & 2014 & Prospective & Did not compare smokers vs. no smokers \\
\hline Kütan et al. [47] & 2015 & Randomized clinical trial & Did not report information on smoking \\
\hline Gomes et al. [48] & 2015 & Prospective & Did not report information on smoking \\
\hline Becker et al. [49] & 2015 & Prospective & Did not report information on smoking \\
\hline Donati et al. [50] & 2015 & Prospective & Did not report information on smoking \\
\hline Pozzi et al. [51] & 2015 & Prospective & Did not report information on peri-implantitis \\
\hline Schwarz et al. [52] & 2015 & Cross sectional & Not prospective \\
\hline Krennmair et al. [53] & 2016 & Prospective & Did not compare smokers vs. no smokers \\
\hline van Velzen et al. [54] & 2015 & Prospective & Did not compare smokers vs. no smokers \\
\hline Malchiodi et al. [55] & 2015 & Prospective & Did not compare smokers vs. no smokers \\
\hline Hita-Iglesias et al. [56] & 2015 & Prospective & Did not report information on peri-implantitis \\
\hline Daubert et al. [57] & 2015 & Cross sectional & Not prospective \\
\hline Gherlone et al. [58] & 2015 & Prospective & Sample not representative of general population \\
\hline Konermann et al. [59] & 2016 & Cross sectional & Not prospective \\
\hline
\end{tabular}

The criteria used to diagnose peri-implantitis and periodontal disease slightly varied among the included studies, one study [31] did not define clear diagnostic criteria for assessing history of chronic periodontitis.

Two studies [32,33] showed a higher and statistically significant implant loss rate in patients with a history of periodontitis both on an implant and patient level; also in the third study [31] the implant loss rate (on an implant level; patient level data were not reported) was greater in periodontal than in healthy patients, but without reaching a statistical significance.

Two studies $[\underline{32}, \underline{33}]$ found a higher and statistically significant peri-implantitis incidence in the periodontally compromised patients groups, both on an implant and patient level, while the third study [31] reached the same conclusions on an implant level only (patient level data not reported).

Detailed data of the included studies are listed in Table 3.

\section{Effect of smoking}

The total number of treated patients in the selected study was 53 [31]. Sex distribution and age of the sample were not reported. The total number of inserted implants was 112 (28 implants in 12 smoker patients, 84 implants in 41 no smoker patients).

The criteria used to diagnose peri-implantitis was the presence of probing depth $\geq 5 \mathrm{~mm}$, associated to bleeding on probing and radiographic signs of bone loss.

In this study, implant loss rate and peri-implantitis incidence, on an implant level, resulted higher in smoker than in no smoker patients, but without reaching a statistical significance (patient level data not reported).

Detailed data of the included study are listed in Table 4. 
Table 3. Characteristics of included studies (history of periodontitis)

\begin{tabular}{|c|c|c|c|c|c|c|c|c|c|c|}
\hline Study & $\begin{array}{c}\text { Year of } \\
\text { publication }\end{array}$ & $\begin{array}{l}\text { Study } \\
\text { design }\end{array}$ & $\begin{array}{l}\text { Patients/ } \\
\text { implants }\end{array}$ & $\begin{array}{l}\text { Mean Age/Sex } \\
\text { (years [M/F] })\end{array}$ & $\begin{array}{l}\text { Implant failures } \\
\text { (events/implants; } \\
\text { events/patients) }\end{array}$ & $\begin{array}{c}\text { Peri-implantitis } \\
\text { (events/implants; } \\
\text { events/patients) }\end{array}$ & $\begin{array}{c}\text { Smokers } \\
\text { Smokers/Non- } \\
\text { smokers }\end{array}$ & $\begin{array}{l}\text { Diagnosis of peri- } \\
\text { implantitis }\end{array}$ & $\begin{array}{c}\text { Diagnosis of } \\
\text { periodontal disease }\end{array}$ & Confounders \\
\hline Karoussis et al. [31] & 2003 & $\begin{array}{c}\text { Prospective } \\
\text { cohort }\end{array}$ & $\begin{array}{l}\text { H: } 45 / 91 \\
\text { CP: } 8 / 21\end{array}$ & $\mathrm{NR}$ & $\begin{array}{l}\mathrm{H}: 3 / 91 ; \mathrm{NR} / 45 \\
\mathrm{CP}: 2 / 21 ; \mathrm{NR} / 8\end{array}$ & $\begin{array}{l}\text { H: } 5 / 91 ; \mathrm{NR} / 45 \\
\text { CP: } 8 / 21 ; \mathrm{NR} / 8\end{array}$ & $28 / 84$ & $\begin{array}{c}\mathrm{PD} \geq 5 \mathrm{~mm}+\mathrm{BOP}+ \\
\text { radiographic signs of } \mathrm{BL}\end{array}$ & NR & $\begin{array}{l}\text { Declared: } \\
\text { smoking, different prosthetic rehabilitations. } \\
\text { Discussed: } \\
\text { smoking. }\end{array}$ \\
\hline Gatti et al. [32] & 2008 & $\begin{array}{l}\text { Prospective } \\
\text { cohort }\end{array}$ & $\begin{array}{l}\text { H: } 29 / 72 \\
\text { MP: } 7 / 26 \\
\text { SP: } 26 / 129\end{array}$ & $\begin{array}{l}\text { H: } 40(9 / 20) \\
\text { MP: } 56(4 / 3) \\
\text { SP: } 56(10 / 16)\end{array}$ & $\begin{array}{l}\text { H: } 0 / 72 ; 0 / 29 \\
\text { MP: } 0 / 26 ; 0 / 7 \\
\text { SP: } 2 / 129 ; 1 / 26\end{array}$ & $\begin{array}{l}\text { H: } 0 / 72 ; 0 / 29 \\
\text { MP: } 0 / 26 ; 0 / 7 \\
\text { SP: } 4 / 129 ; 2 / 26\end{array}$ & $\begin{array}{l}\text { 14/48 } \\
\text { H: }(6 / 29) \\
\text { MP: }(3 / 26) \\
\text { SP: }(5 / 26)\end{array}$ & $\begin{array}{l}\mathrm{PD} \geq 5 \mathrm{~mm}+\text { suppuration } \\
\text { or other signs of infection } \\
\quad+\mathrm{BL}>2 \mathrm{~mm}\end{array}$ & $\begin{array}{l}\text { Periodontal screening and } \\
\text { recording index: } 0-2(\mathrm{H}) 3 \\
\text { (MP) or } 4 \text { (SP) }\end{array}$ & $\begin{array}{l}\text { Declared: } \\
\text { age, smoking, regenerative procedures, healing period, prosthetic rehabilitations, } \\
\text { implant brands, oral hygiene maintenance. } \\
\text { Discussed: } \\
\text { age, smoking, implant brands. }\end{array}$ \\
\hline Swierkot et al. [33] & 2012 & $\begin{array}{l}\text { Prospective } \\
\text { cohort }\end{array}$ & $\begin{array}{c}\text { H: } 18 / 30 \\
\text { GaP: } 35 / 149\end{array}$ & $\begin{array}{l}\text { GaP: } 39.6(15 / 20) \\
\text { H: } 38.6(9 / 9)\end{array}$ & $\begin{array}{c}\text { H: } 0 / 30 ; 0 / 18 \\
\text { GaP: } 6 / 149 ; 3 / 35\end{array}$ & $\begin{array}{c}\text { H: } 3 / 30 ; 2 / 18 \\
\text { GaP: } 39 / 149 ; 15 / 35\end{array}$ & $14 / 39$ & $\begin{array}{c}\mathrm{PD} \geq 5 \mathrm{~mm}+\mathrm{BOP}+\mathrm{BL} \\
\quad>0,2 \mathrm{~mm} / \text { years }\end{array}$ & According to AAP criteria & $\begin{array}{l}\text { Declared: } \\
\text { smoking, sex, age, implant features, prosthetic rehabilitations, bone quality, } \\
\text { regenerative procedures. } \\
\text { Discussed: } \\
\text { smoking, sex, age, implant features, prosthetic rehabilitation, bone quality, } \\
\text { regenerative procedures. }\end{array}$ \\
\hline
\end{tabular}

$\mathrm{M}=$ male; $\mathrm{F}=$ female; $\mathrm{H}=$ healthy; $\mathrm{CP}=$ chronic periodontitis; $\mathrm{MP}=$ moderate periodontitis; $\mathrm{SP}=$ severe periodontitis; $\mathrm{GaP}$ = generalised aggressive periodontitis; $\mathrm{PD}=$ probing depth; $\mathrm{BOP}=$ bleeding on probing; $\mathrm{BL}=$ bone loss; $\mathrm{NR}=$ not reported.

Table 4. Characteristics of the included study (smoking habits)

\begin{tabular}{c|c|c|c|c|c|c|c|c}
\hline \multicolumn{1}{c|}{ Study } & Study design & $\begin{array}{c}\text { Patients/ } \\
\text { implants }\end{array}$ & $\begin{array}{c}\text { gge/ } \\
\text { sex }\end{array}$ & $\begin{array}{c}\text { Implant failures } \\
\text { (events/implants) }\end{array}$ & $\begin{array}{c}\text { Peri-implantitis } \\
\text { (events/implants) }\end{array}$ & $\begin{array}{c}\text { Diagnosis of peri- } \\
\text { implantitis }\end{array}$ & $\begin{array}{c}\text { Definition of } \\
\text { smoking }\end{array}$ & \multicolumn{1}{c}{ Confounders } \\
\hline Karoussis et al. [31] & Prospective cohort & $\begin{array}{c}\text { Smokers }=12 / 18 \\
\text { Non-smokers }=41 / 84\end{array}$ & NR & $\begin{array}{c}\text { Smokers (2/28) } \\
\text { Non-smokers (3/84) }\end{array}$ & $\begin{array}{c}\text { Smokers (5/28) } \\
\text { Non-smokers (8/84) }\end{array}$ & $\begin{array}{c}\text { PD } \geq 5 \mathrm{~mm}+\mathrm{BOP}+ \\
\text { radiographic signs of BL }\end{array}$ & $\begin{array}{l}\text { Declared: } \\
\text { history of periodontitis, different prosthetic rehabilitations. } \\
\text { Niscussed: } \\
\text { history of periodontitis. }\end{array}$ \\
\hline
\end{tabular}

$\mathrm{PD}=$ probing depth; $\mathrm{BOP}=$ bleeding on probing; $\mathrm{BL}=$ bone loss; $\mathrm{NR}=$ not reported.

\begin{tabular}{|c|c|c|c|c|c|c|c|c|c|}
\hline \multirow[b]{2}{*}{ Study or Subgroup } & \multicolumn{2}{|c|}{ Healthy } & \multicolumn{2}{|c|}{ Periodontitis } & \multirow{2}{*}{ Weight } & \multirow{2}{*}{$\begin{array}{c}\text { Risk Ratio } \\
\text { M-H, Random, 95\% Cl }\end{array}$} & & \multirow{2}{*}{\multicolumn{2}{|c|}{$\begin{array}{c}\text { Risk Ratio } \\
\text { M-H, Random, } 95 \% \mathrm{Cl}\end{array}$}} \\
\hline & Events $T$ & Total & Events & Total & & & & & \\
\hline Karoussis et al. [31] & 3 & 91 & 2 & 21 & $59.1 \%$ & $0.35[0.06,1.94]$ & & & \\
\hline Gatti et al. [32] & 0 & 72 & 2 & 155 & $19.2 \%$ & $0.43[0.02,8.79]$ & & & \\
\hline Swierkot et al. [33] & 0 & 30 & 6 & 149 & $21.7 \%$ & $0.37[0.02,6.44]$ & & & \\
\hline Total $(95 \% \mathrm{Cl})$ & & 193 & & 325 & $100 \%$ & $0.37[0.1,1.38]$ & & & \\
\hline \multirow{2}{*}{\multicolumn{7}{|c|}{$\begin{array}{l}\text { Total events } \\
\text { Heterogeneity: } \text { Tau }^{2}=0 ; \mathrm{Chi}^{2}=0.01, \mathrm{df}=2 \quad 10 \\
\text { Test for overall effect: } Z=1.48(P=0.14)\end{array}$}} & & & \\
\hline & & & & & & & 0.01 & $\begin{array}{l}0.1 \\
\text { Favours [Healthy] }\end{array}$ & $1{ }^{1}$ Favours [Periodontitis] 100 \\
\hline
\end{tabular}

Figure 2. Forest plot for the event 'implant loss' comparing periodontally healthy with periodontally compromised patients (implant as statistical unit).

\begin{tabular}{|c|c|c|c|c|c|c|c|c|c|}
\hline Study or Subgroup & \multicolumn{2}{|c|}{$\begin{array}{l}\text { Healthy } \\
\text { Events Total }\end{array}$} & \multicolumn{2}{|c|}{ Periodontitis } & Weight & $\begin{array}{c}\text { Risk Ratio } \\
\text { M-H, Random, 95\% Cl }\end{array}$ & & \multicolumn{2}{|c|}{$\begin{array}{l}\text { Risk Ratio } \\
\text { M-H, Random, 95\% CI }\end{array}$} \\
\hline Karoussis et al. [31] & 5 & 91 & 8 & 21 & $51.1 \%$ & $0.14[0.05,0.4]$ & & $=$ & \\
\hline Gatti et al. [32] & 0 & 72 & 4 & 155 & $6.2 \%$ & $0.24[0.01,4.35]$ & & & \\
\hline Swierkot et al. [33] & 3 & 30 & 39 & 149 & $42.7 \%$ & $0.38[0.13,1.16]$ & & & \\
\hline Total $(95 \%$ Cl) & & 193 & & 325 & $100 \%$ & $0.23[0.11,0.46]$ & & & \\
\hline \multirow{2}{*}{\multicolumn{6}{|c|}{$\begin{array}{l}\text { Total events } \\
\text { Heterogeneity: } \text { Tau }^{2}=0 ; \mathrm{Chi}^{2}=1.78, \mathrm{df}=2(\mathrm{P}=0.41) ; \mathrm{I}^{2}=0 \% \\
\text { Test for overall effect: } Z=4.04(P<0.0001)\end{array}$}} & & & & \\
\hline & & & & & & & 0.01 & $\begin{array}{c}0.1 \\
\text { Favours [Healthy] }\end{array}$ & $\begin{array}{c}10 \\
1 \\
\text { Favours [Periodontitis] }\end{array}$ \\
\hline
\end{tabular}

Figure 3. Forest plot for the event 'peri-implantitis' comparing periodontally healthy with periodontally compromised patients (implant as statistical unit).

\begin{tabular}{|c|c|c|c|c|c|c|c|c|c|}
\hline \multirow{3}{*}{$\begin{array}{l}\text { Study or Subgroup } \\
\text { Gatti et al. [32] } \\
\text { Swierkot et al. [33] }\end{array}$} & \multicolumn{2}{|c|}{$\begin{array}{l}\text { Healthy } \\
\text { Events Total }\end{array}$} & \multicolumn{2}{|c|}{$\begin{array}{l}\text { Periodontitis } \\
\text { Events Total }\end{array}$} & Weight & \multirow{2}{*}{$\begin{array}{c}\begin{array}{c}\text { Risk Ratio } \\
\text { M-H, Random, } 95 \% \mathrm{Cl}\end{array} \\
0.23[0.01,4.54]\end{array}$} & \multicolumn{3}{|c|}{$\begin{array}{c}\text { Risk Ratio } \\
\text { M-H, Random, } 95 \% \mathrm{Cl} \\
\end{array}$} \\
\hline & 0 & 29 & 2 & 33 & $17.1 \%$ & & & & \\
\hline & 2 & 18 & 15 & 35 & $82.9 \%$ & $0.26[0.07,1.01]$ & & & \\
\hline Total $(95 \%$ Cl) & & 47 & & 68 & $100 \%$ & $0.25[0.07,0.88]$ & & & \\
\hline \multirow{2}{*}{\multicolumn{6}{|c|}{$\begin{array}{l}\text { Total events } \\
\text { Heterogeneity: Tau' }=0 ; \mathrm{Chi}^{2}=0.01, \mathrm{df}=1 \quad 17 \\
\text { Test for overall effect: } Z=2.17(P=0.94) ; l^{2}=0 \%\end{array}$}} & & & & \\
\hline & & & & & & & 0.01 & $\begin{array}{c}0.1 \\
\text { Favours [Healthy] }\end{array}$ & ]$^{1} \begin{array}{c}10 \\
\text { Favours [Periodontitis] }\end{array}$ \\
\hline
\end{tabular}

Figure 4. Forest plot for the event 'implant loss' comparing periodontally healthy with periodontally compromised patients (patient as statistical unit).

\begin{tabular}{|c|c|c|c|c|c|c|c|c|c|}
\hline \multirow{3}{*}{$\begin{array}{l}\text { Study or Subgroup } \\
\text { Gatti et al. [32] } \\
\text { Swierkot et al. [33] }\end{array}$} & \multicolumn{2}{|c|}{$\begin{array}{l}\text { Healthy } \\
\text { Events Total }\end{array}$} & \multicolumn{2}{|c|}{ Periodontitis } & Weight & \multirow{3}{*}{$\begin{array}{c}\begin{array}{c}\text { Risk Ratio } \\
\text { M-H, Random, 95\% Cl }\end{array} \\
0.38[0.02,8.93] \\
0.27[0.01,4.97]\end{array}$} & \multicolumn{3}{|c|}{$\begin{array}{l}\text { Risk Ratio } \\
\text { M-H, Random, } 95 \% \mathrm{Cl} \\
\end{array}$} \\
\hline & 0 & 29 & 1 & 33 & $45.9 \%$ & & & $=$ & \\
\hline & 0 & 18 & 3 & 35 & $54.1 \%$ & & & & \\
\hline Total $(95 \% \mathrm{Cl})$ & & 47 & & 68 & $100 \%$ & $0.32[0.04,2.69]$ & & & \\
\hline \multirow{2}{*}{\multicolumn{6}{|c|}{$\begin{array}{l}\text { Teterogeneity: } \text { Tau }^{2}=0 ; \mathrm{Chi}^{2}=0.02, \text { df }=1(P=0.88) ; L^{2}=0 \% \\
\text { Test for overall effect: } Z=1.06(P=0.29)\end{array}$}} & & & & \\
\hline & & & & & & & 0.01 & $\begin{array}{c}0.1 \\
\text { Favours [Healthy] }\end{array}$ & 1 Favours [Periodontitis] 100 \\
\hline
\end{tabular}

Figure 5. Forest plot for the event 'peri-implantitis' comparing periodontally healthy with periodontally compromised patients (patient as statistical unit).

J Oral Maxillofac Res 2016 (Jul-Sep) | vol. 7 | No 3 | e3 | p. 7 (page number not for citation purposes) 


\section{Results of meta-analysis}

Meta-analysis could be performed only for the three studies analysing the influence of history of periodontal disease: detailed results for the primary outcomes are shown in Figures 2 - 5. The RRs are expressed as mean $(95 \% \mathrm{CI})$.

For the implant loss (considering the implant as the statistical unit) the overall RR was 0.37 (0.1 - 1.38) in favour of the periodontally healthy patients. The overall effect was not significant $(\mathrm{P}=0.14)$ (Figure 2). For the occurrence of peri-implantitis (considering the implant as the statistical unit), the overall RR was $0.23(0.11-0.46)$ in favour of the periodontally healthy patients. The overall effect was significant $(\mathrm{P}$ $<0.0001$ ) (Figure 3). For the implant loss (considering the patient as the statistical unit), the overall RR was $0.32(0.04-2.69)$ in favour of the periodontally healthy patients. The overall effect was not significant $(\mathrm{P}=0.29)$ (Figure 4). For the occurrence of periimplantitis (considering the patient as the statistical unit), the overall RR was $0.25(0.07-0.88)$ in favour of the periodontally healthy patients. The overall effect was significant $(\mathrm{P}=0.003)$ (Figure 5).

In all the comparisons, no heterogeneity was seen with $\mathrm{I}^{2}=0 \%$ and Chi square not significant $(\mathrm{P}=0.41$, at least).

\section{Risk of bias in individual studies}

None of the included studies reached the maximum score of the NOS (Tables 5 and 6). Two studies $[31,32]$ reached the maximum score for the selection item, one study [32] reached the maximum score for the exposure item, and no study had the maximum score for the comparability item.

\section{Additional analysis}

Due to the limited number of included studies, additional investigations regarding publication bias or sensitivity analysis were not performed.

\section{DISCUSSION}

When analysing the risk factors of a disease, a clear definition of the indagated pathological condition is of paramount importance. Then, especially in multifactorial pathologies, an accurate stratification of the sample and a strict control of the various confounding factors are crucial points to design appropriate trials, in order to evaluate the impact of each single risk factor on the onset of the disease. In literature, a variety of different parameters and diagnostic criteria have been used when studying incidence, prevalence and risk factors of periimplant diseases: in a recent review on the quality of the clinical research, Tomasi and Derks [60] indicate the absolute need for improved reporting of epidemiological studies on this topic.

We found a confirmation of this statement in our systematic review, whose aim was to verify whether history of periodontitis and smoking habits may act as predisposing factors for implant loss and occurrence of peri-implantitis. In fact, a very limited number of articles could be included in the final analysis, seeming to confirm the influence of the history of periodontal disease as a potentially relevant risk factor for the onset of peri-implantitis, analysed both at implant and at patient level. However, history of periodontitis did not result as a significant risk factor for implant loss. It was impossible to draw general conclusions regarding the impact of smoking in favouring peri-implantitis and implant loss because only one study could be selected for the qualitative analysis.

The results of our meta-analysis are consistent with the conclusions of previous systematic reviews examining the history of periodontal disease as a risk factor for the onset of peri-implantitis, but applying broader inclusion criteria [ $\underline{7-9}, \underline{61}]$.

Peri-implantitis is actually defined as an inflammatory condition with bleeding on probing and/or purulent exudate, associated with clinically significant progressive

Table 5. Risk of bias in the included studies (history of periodontitis)

\begin{tabular}{l|c|c|c|c|c|c|c|c|c}
\hline & \multicolumn{4}{|c|}{ Selection } & Comparability & \multicolumn{4}{c}{ Outcome } \\
\cline { 2 - 10 } & $\mathbf{1}$ & $\mathbf{2}$ & $\mathbf{3}$ & $\mathbf{4}$ & $\mathbf{1}$ & $\mathbf{1}$ & $\mathbf{2}$ & $\mathbf{3}$ & Total \\
\hline Karoussis et al. [31] & $\star \mathrm{b}$ & $\star$ & $\star \mathrm{a}$ & $\star$ & & $\star \mathrm{b}$ & $\star$ & & $6 \star$ \\
\hline Gatti et al. [32] & $\star \mathrm{b}$ & $\star$ & $\star \mathrm{a}$ & $\star$ & & $\star \mathrm{b}$ & $\star$ & $\star \mathrm{b}$ & $7 \star$ \\
\hline Swierkot et al. [33] & $\star \mathrm{b}$ & & $\star \mathrm{a}$ & $\star$ & & $\star \mathrm{b}$ & $\star$ & & $5 \star$ \\
\hline
\end{tabular}

Table 6. Risk of bias in the included study (smoking habits)

\begin{tabular}{c|c|c|c|c|c|c|c|c|c}
\hline & \multicolumn{4}{|c|}{ Selection } & Comparability & \multicolumn{4}{c}{ Outcome } \\
\cline { 2 - 10 } & $\mathbf{1}$ & $\mathbf{2}$ & $\mathbf{3}$ & $\mathbf{4}$ & $\mathbf{1}$ & $\mathbf{1}$ & $\mathbf{2}$ & $\mathbf{3}$ & Total \\
\hline Karoussis et al. [31] & $\star \mathrm{b}$ & $\star$ & $\star \mathrm{a}$ & $\star$ & & $\star \mathrm{b}$ & $\star$ & & $6 \star$ \\
\hline
\end{tabular}


crestal bone loss after the adaptive phase [62-64]. The presence of bone loss and probing depth alone may not be enough to formulate a diagnosis of periimplantitis: bone loss can have a number of nonbacterial causes including surgical technique [65], implant design [66], implant positioning [67], crestal bone thickness $[68,69]$ or occlusal overload [70]. For this reason, we excluded a consistent number of studies (although included in recent reviews $[7-9,61, \underline{71}, \underline{72}])$, which were reporting only data concerning bone loss around implants, without giving a definite diagnosis of peri-implantitis.

According to Lindhe and Meyle [62], who stated that the assessment of incidence and risk factors require longitudinal, prospective or case-control studies, we included in the present review only prospective trials comparing a cohort of individuals presenting the investigated risk factor with a group of not exposed individuals in the format of a longitudinal study. However, it is necessary to evaluate prospective cohort studies for internal validity due to the presence of selection bias and confounding variables. The three publications included in this systematic review [31-33] did not fully address the STROBE checklist for cohort studies, especially in terms of sample size definition, exposures, predictors, potential confounders, and effect modifiers. Also the diagnostic criteria for peri-implantitis were slightly different among the studies and the definition of periodontal disease followed different parameters (one study did not report it at all [31]). Potential confounders (e.g. systemic conditions, age, sex, bone quality, implant features, type of prosthesis, parafunctions) were only partially reported and seldom assessed during outcomes analysis. The quality assessment following NOS (Newcastle-Ottawa Scale) scheme, accordingly, revealed the absence of complete adherence to the different items.

\section{Summary of evidence}

This systematic review reported weak evidence supporting the hypothesis that history of periodontal disease could act as a risk factor for peri-implantitis; moreover, no evidence was found to clarify the influence of smoking in the pathogenesis of this disease. History of periodontitis resulted to be a risk factor for the occurrence of peri-implantitis both in patient and in implant-based analyses, but it did not result a significant risk factor for implant loss. However, according to the low number of selected studies and their limitations (for both the analysed issues), no clinical recommendations could be given. Further prospective trials are needed to confirm the results of the present meta-analysis: future studies should include adjustment of their results for known and potential confounding factors, and should report their results according to a clear and widely accepted definition of peri-implantitis, together with a well-defined stratification of periodontal disease and smoking status.

\section{Limitations}

It is worth considering that the narrow inclusion criteria adopted in this review increase studies homogeneity but, at the same time, also the risk to exclude significant data [73]: nevertheless, we think that this strict methodological approach may help readers to understand the real available evidence on this specific topic, with all the limitations and drawbacks, and could be a motivation for researchers in designing appropriate trials.

Hence, the results reported in this review should be interpreted with great caution. The low number of studies, the heterogeneity of case definitions and the substantial lack of control of the confounders represent concrete risks of bias for the correct estimation of the role of smoking habits and history of periodontitis in favouring the onset of peri-implant diseases.

\section{CONCLUSIONS}

Based on the results of the present systematic review to assess the influence of smoking habits and history of periodontal disease as risk factors for implant loss and occurrence of peri-implantitis, the following conclusions can be drawn:

1. There is limited evidence to suggest that history of periodontal disease could be a risk factor for peri-implantitis;

2. There are no sufficient data to assess the role of smoking as a risk factor for implant loss and periimplantitis.

Adequately powered long-term prospective studies are necessary to analyse the role of these potential risk factors using clear and accepted disease definitions, accurate sample selection and strict control of confounders.

\section{ACKNOWLEDGMENTS AND DISCLOSURE STATEMENTS}

The authors have no conflicts of interest to disclose. The study was self-funded. 


\section{RFERENCES}

1. Jung RE, Pjetursson BE, Glauser R, Zembic A, Zwahlen M, Lang NP. A systematic review of the 5-year survival and complication rates of implant-supported single crowns. Clin Oral Implants Res. 2008 Feb;19(2):119-30. [Medline: 18067597] [doi: 10.1111/j.1600-0501.2007.01453.x]

2. Pjetursson BE, Tan K, Lang NP, Brägger U, Egger M, Zwahlen M. A systematic review of the survival and complication rates of fixed partial dentures (FDPs) after an observation period of at least 5 years. Clin Oral Implants Res. 2004 Dec;15(6):625-42. [Medline: 15533124] [doi: 10.1111/j.1600-0501.2004.01117.x]

3. Ong CT, Ivanovski S, Needleman IG, Retzepi M, Moles DR, Tonetti MS, Donos N. Systematic review of implant outcomes in treated periodontitis subjects. J Clin Periodontol. 2008 May;35:438-62. [Medline: 18433385] [doi: 10.1111/j.1600-051X.2008.01207.x]

4. Ferreira SD, Silva GL, Cortelli JR, Costa JE, Costa FO. Prevalence and risk variables for peri-implant disease in Brazilian subjects. J Clin Periodontol. 2006 Dec;33(12):929-35. [Medline: 17092244] [doi: 10.1111/j.1600-051X.2006.01001.x]

5. Roccuzzo M, De Angelis N, Bonino L, Aglietta M. Ten-year results of a three-arm prospective cohort study on implants in periodontally compromised patients. Part 1: implant loss and radiographic bone loss. Clin Oral Implants Res. 2010 May;21(5):490-6. [Medline: 20337668] [doi: 10.1111/j.1600-0501.2009.01886.x]

6. Konstantinidis IK, Kotsakis GA, Gerdes S, Walter MH. Cross-sectional study on the prevalence and risk indicators of peri-implant diseases. Eur J Oral Implantol. 2015 Spring;8(1):75-88. [Medline: 25738181]

7. Safii SH, Palmer RM, Wilson RF. Risk of implant failure and marginal bone loss in subjects with a history of periodontitis: a systematic review and meta-analysis. Clin Implant Dent Relat Res. 2010 Sep;12(3):165-74. [Medline: 19438942]

8. Chrcanovic BR, Albrektsson T, Wennerberg A. Periodontally compromised vs. periodontally healthy patients and dental implants: a systematic review and meta-analysis. J Dent. 2014 Dec;42(12):1509-27. [Medline: 25283479] [doi: 10.1016/j.jdent.2014.09.013]

9. Sgolastra F, Petrucci A, Severino M, Gatto R, Monaco A. Periodontitis, implant loss and peri-implantitis. A meta-analysis. Clin Oral Implants Res. 2015 Apr;26(4):e8-16. [Medline: 24382358] [doi: 10.1111/clr.12319]

10. Turri A, Rossetti PH, Canullo L, Grusovin MG, Dahlin C. Prevalence of peri-implantitis in medically compromised patients and smokers: a systematic review. Int J Oral Maxillofac Implants. 2016 Jan-Feb;31(1):111-8. [Medline: 26800167] [doi: 10.11607/jomi.4149]

11. Liberati A, Altman DG, Tetzlaff J, Mulrow C, Gøtzsche PC, Ioannidis JP, Clarke M, Devereaux PJ, Kleijnen J, Moher D. The PRISMA statement for reporting systematic reviews and meta-analyses of studies that evaluate health care interventions: explanation and elaboration. J Clin Epidemiol. 2009 Oct;62(10):e1-34. [Medline: 19631507] [doi: 10.1016/j.jclinepi.2009.06.006]

12. Landis JR, Koch GG. The measurement of observer agreement for categorical data. Biometrics. 1977 Mar;33(1):159-74. [Medline: 843571$]$ [doi: $10.2307 / 2529310]$

13. Wells GA, Shea B, O’Connell D, Peterson J, Welch V, Losos M, Tugwell P. The NewcastleOttawa Scale (NOS) for assessing the quality of nonrandomised studies in meta-analyses. [URL: http://www.ohri.ca/programs/clinical_epidemiology/oxford.htm]

14. Lau J, Ioannidis JP, Schmid CH. Quantitative synthesis in systematic reviews. Ann Intern Med. 1997 Nov;127(9):820-6. [Medline: 9382404] [doi: 10.7326/0003-4819-127-9-199711010-00008]

15. Higgins JP, Thompson SG. Quantifying heterogeneity in a meta-analysis. Stat Med. 2002 Jun;21(11):1539-58. [Medline: 12111919 ] [doi: $\underline{10.1002 / \operatorname{sim} .1186]}$

16. Borenstein M, Hedges LV, Higgins JPT, Rothstein HR. In: Introduction to Meta-analysis. Chichester, UK: John Wiley \& Sons, Ltd; 2009. [doi: 10.1002/9780470743386]

17. Sbordone L, Barone A, Ciaglia RN, Ramaglia L, Iacono VJ. Longitudinal study of dental implants in a periodontally compromised population. J Periodontol. 1999 Nov;70(11):1322-9. [Medline: 10588495] [doi: 10.1902/jop.1999.70.11.1322]

18. Mengel R, Schroeder T, Flores-de-Jacoby L. Osseointegrated implants in patients treated for generalized chronic periodontitis and generalized aggressive periodontitis: 3 - and 5-year results of a prospective long-term study. J Periodontol. 2001 Aug;72(8):977-89. [Medline: 11525449] [doi: 10.1902/jop.2001.72.8.977]

19. Mengel R, Flores-de-Jacoby L. Implants in patients treated for generalized aggressive and chronic periodontitis: a 3-year prospective longitudinal study. J Periodontol. 2005 Apr;76(4):534-43. [Medline: 15857093] [doi: 10.1902/jop.2005.76.4.534]

20. Mengel R, Behle M, Flores-de-Jacoby L. Osseointegrated implants in subjects treated for generalized aggressive periodontitis: 10-year results of a prospective, long-term cohort study. J Periodontol. 2007 Dec;78(12):2229-37. [Medline: 18052693] [doi: 10.1902/jop.2007.070201]

21. Fardal Ø, Linden GJ. Tooth loss and implant outcomes in patients refractory to treatment in a periodontal practice. J Clin Periodontol. 2007 Aug;35(8):733-8. [Medline: 18498381] [doi: 10.1111/j.1600-051X.2008.01247.x] 
22. De Boever AL, Quirynen M, Coucke W, Theuniers G, De Boever JA. Clinical and radiographic study of implant treatment outcome in periodontally susceptible and non-susceptible patients: a prospective long-term study. Clin Oral Implants Res. 2009 Dec;20(12):1341-50. [Medline: 19793321] [doi: 10.1111/j.1600-0501.2009.01750.x]

23. Koldsland OC, Scheie AA, Aass AM. Prevalence of implant loss and the influence of associated factors. J Periodontol. 2009 Jul;80(7):1069-75. [Medline: 19563286] [doi: 10.1902/jop.2009.080594]

24. Levin L, Ofec R, Grossmann Y, Anner R. Periodontal disease as a risk for dental implant failure over time: a long-term historical cohort study. J Clin Periodontol. 2011 Aug;38(8):732-7. [Medline: 21635280] [doi: 10.1111/j.1600-051X.2011.01745.x]

25. Horwitz J, Machtei EE. Immediate and delayed restoration of dental implants in patients with a history of periodontitis: a prospective evaluation up to 5 years. Int J Oral Maxillofac Implants. 2012 Sept-Oct;27(5):1137-43. [Medline: 23057027]

26. Pjetursson BE, Helbling C, Weber HP, Matuliene G, Salvi GE, Brägger U, Schmidlin K, Zwahlen M, Lang NP. Peri-implantitis susceptibility as it relates to periodontal therapy and supportive care. Clin Oral Implants Res. 2012 Jul;23(7):888-94. [Medline: 22530771] [doi: 10.1111/j.1600-0501.2012.02474.x]

27. Casado PL, Pereira MC, Duarte ME, Granjeiro JM. History of chronic periodontitis is a high risk indicator for periimplant disease. Braz Dent J. 2013 Mar-Apr;24(2):136-41. [Medline: 23780361] [doi: 10.1590/0103-6440201302006]

28. Jiang BQ, Lan J, Huang HY, Liang J, Ma XN, Huo LD, Xu X. A clinical study on the effectiveness of implant supported dental restoration in patients with chronic periodontal diseases. Int J Oral Maxillof Surg. 2013 Feb;42(2):256-9. [Medline: 23021323] [doi: 10.1016/j.ijom.2012.08.001]

29. Marrone A, Lasserre J, Bercy P, Brecx MC. Prevalence and risk factors for peri-implant disease in Belgian adults. Clin Oral Implants Res. 2013 Aug;24(8):934-40. [Medline: 22551347] [doi: 10.1111/j.1600-0501.2012.02476.x]

30. Roccuzzo M, Bonino L, Dalmasso P, Aglietta M. Long-term results of a three arms prospective cohort study on implants in periodontally compromised patients: 10-year data around sandblasted and acid-etched (SLA) surface. Clin Oral Implants Res. 2014 Oct;25(10):1105-12. [Medline: 23865554] [doi: 10.1111/clr.12227]

31. Karoussis IK, Salvi GE, Heitz-Mayfield LJ, Brägger U, Hämmerle CH, Lang NP. Long-term implant prognosis in patients with and without a history of chronic periodontitis: a 10- year prospective cohort study of the ITI Dental Implant System. Clin Oral Implants Res. 2003 Jun;14(3):329-39. [Medline: 12755783] [doi: 10.1034/j.1600-0501.000.00934.x]

32. Gatti C, Gatti F, Chiapasco M, Esposito M. Outcome of dental implants in partially edentulous patients with and without a history of periodontitis: a 5-year interim analysis of a cohort study. Eur J Oral Implantol. 2008 Spring;1(1):45-51. [Medline: 20467643]

33. Swierkot K, Lottholz P, Flores-de-Jacoby L, Mengel R. Mucositis, peri-implantitis, implant success, and survival of implants in patients with treated generalized aggressive periodontitis: 3 - to 16-year results of a prospective long-term cohort study. J Periodontol. 2012 Oct;83(10):1213-25. [Medline: 22264211] [doi: 10.1902/jop.2012.110603]

34. Roos-Jansåker AM, Renvert H, Lindahl C, Renvert S. Nine- to fourteen-year follow-up of implant treatment. Part III: factors associated with peri-implant lesions. J Clin Periodontol. 2006 Apr;33(4):296-301. [Medline: 16553639] [doi: 10.1111/j.1600-051X.2006.00908.x]

35. Costa FO, Takenaka-Martinez S, Cota LO, Ferreira SD, Silva GL, Costa JE. Peri-implant disease in subjects with and without preventive maintenance: a 5-year follow-up. J Clin Periodontol. 2012 Feb;39(2):173-81. [Medline: 22111654] [doi: 10.1111/j.1600-051X.2011.01819.x]

36. Dvorak G, Arnhart C, Heuberer S, Huber CD, Watzek G, Gruber R. Peri-implantitis and late implant failures in postmenopausal women: a cross-sectional study. J Clin Periodontol. 2011 Oct;38(10): 950-5. [Medline: 21777269] [doi: 10.1111/j.1600-051X.2011.01772.x]

37. Schropp L, Wenzel A, Stavropoulos A. Early, delayed, or late single implant placement: 10-year results from a randomized controlled clinical trial. Clin Oral Implants Res. 2014 Dec;25(12):1359-65. [Medline: 25040354] [doi: $10.1111 /$ clr.12273]

38. Göthberg C, André U, Gröndahl K, Ljungquist B, Thomsen P, Slotte C. Immediately loaded implants with or without abutments supporting fixed partial dentures: 1-year results from a prospective, randomized, clinical trial. Clin Implant Dent Relat Res. 2014 Aug;16(4):487-500. [Medline: 21777269] [doi: 10.1111/cid.12025]

39. Sanz M, Ivanoff CJ, Weingart D, Wiltfang J, Gahlert M, Cordaro L, Ganeles J, Brägger U, Jackowski J, Martin WC, Jung RE, Chen S, Hämmerle C. Clinical and radiologic outcomes after submerged and transmucosal implant placement with two-piece implants in the anterior maxilla and mandible: 3-year results of a randomized controlled clinical trial. Clin Implant Dent Relat Res. 2015 Apr;17(2):234-46. [Medline: 23837502] [doi: 10.1111/cid.12107]

40. Cecchinato D, Parpaiola A, Lindhe J. Mucosal inflammation and incidence of crestal bone loss among implant patients: a 10-year study. Clin Oral Implants Res. 2014 Jul;25(7):791-6. [Medline: 23763507] [doi: $10.1111 /$ clr.12209]

41. Wang R, Zhao W, Tang ZH, Jin LJ, Cao CF. Peri-implant conditions and their relationship with periodontal conditions in Chinese patients: a cross-sectional study. Clin Oral Implants Res. 2014 Mar;25(3):372-7. [Medline: 23421420] [doi: 10.1111/clr.12114]

42. Meijer HJ, Raghoebar GM, de Waal YC, Vissink A. Incidence of peri-implant mucositis and peri-implantitis in edentulous patients with an implant-retained mandibular overdenture during a 10-year follow-up period. J Clin Periodontol. 2014 Dec;41(12):1178-83. [Medline: 23421420] [doi: 10.1111/jepe.12311] 
43. Canullo L, Pe-arrocha-Oltra D, Covani U, Botticelli D, Serino G, Penarrocha M. Clinical and microbiological findings in patients with peri-implantitis: a cross-sectional study. Clin Oral Implants Res. 2016 Mar;27(3):376-82. [Medline: 25622536] [doi: 10.1111/clr.12557]

44. Aguilar-Salvatierra A, Calvo-Guirado JL, González-Jaranay M, Moreu G, Delgado-Ruiz RA, Gómez-Moreno G. Peri-implant evaluation of immediately loaded implants placed in esthetic zone in patients with diabetes mellitus type 2: a two-year study. Clin Oral Implants Res. 2016 Feb;27(2):156-61. [Medline: 25623884] [doi: $10.1111 / \mathrm{clr} .12552]$

45. Serino G, Turri A, Lang NP. Maintenance therapy in patients following the surgical treatment of periimplantitis: a 5-year follow-up study. Clin Oral Implants Res. 2015 Aug;26(8):950-6. [Medline: 24861154] [doi: 10.1111/clr.12418]

46. Mangano F, Macchi A, Caprioglio A, Sammons RL, Piattelli A, Mangano C. Survival and complication rates of fixed restorations supported by locking-taper implants: a prospective study with 1 to 10 years of follow-up. J Prosthodont. 2014 Aug;23(6):434-44. [Medline: 24750435] [doi: 10.1111/jopr.12152]

47. Kütan E, Bolukbasi N, Yildirim-Ondur E, Ozdemir T. Clinical and radiographic evaluation of marginal bone changes around platform-switching implants placed in crestal or subcrestal positions: a randomized controlled clinical trial. Clin Implant Dent Relat Res. 2015 Oct;17(Suppl 2):e364-75. [Medline: 25041252] [doi: 10.1111/cid.12248]

48. Gomes SC, Corvello P, Romagna R, Müller LH, Angst PD, Oppermann RV. How do peri-implant mucositis and gingivitis respond to supragingival biofilm control - an intra-individual longitudinal cohort study. Eur J Oral Implantol. 2015 Spring;8(1):65-73. [Medline: 25738180]

49. Becker W, Hujoel P, Becker BE, Wohrle P. Dental implants in an aged population: evaluation of periodontal health, bone loss, implant survival, and quality of life. Clin Implant Dent Relat Res. 2015 Jun 17. [Medline: 26082299] [doi: $10.1111 /$ cid.12340]

50. Donati M, Ekestubbe A, Lindhe J, Wennström JL. Implant-supported single-tooth restorations. A 12-year prospective study. Clin Oral Implants Res. 2015 Nov 18. [Medline: 26577573] [doi: 10.1111/clr.12726]

51. Pozzi A, Tallarico M, Moy PK. Immediate loading with a novel implant featured by variable-threaded geometry, internal conical connection and platform shifting: Three-year results from a prospective cohort study. Eur J Oral Implantol. 2015 Spring;8(1):51-63. [Medline: 25738179]

52. Schwarz F, Becker K, Sahm N, Horstkemper T, Rousi K, Becker J. The prevalence of peri-implant diseases for two-piece implants with an internal tube-in-tube connection: a cross-sectional analysis of 512 implants. Clin Oral Implants Res. 2015 Jul 14. [Medline: 26178415] [doi: 10.1111/clr.12609]

53. Krennmair S, Weinländer M, Forstner T, Krennmair G, Stimmelmayr M. Factors affecting peri-implant bone resorption in 4 Implant supported mandibular full-arch restorations: a 3-year prospective study. J Clin Periodontol. 2016 Jan;43(1): 92-101. [Medline: 26445457] [doi: 10.1111/jcpe.12469]

54. van Velzen FJ, Ofec R, Schulten EA, Ten Bruggenkate CM. 10-year survival rate and the incidence of periimplant disease of 374 titanium dental implants with a SLA surface: a prospective cohort study in 177 fully and partially edentulous patients. Clin Oral Implants Res. 2015 Oct;26(10):1121-8. [Medline: 25370914] [doi: 10.1111/clr.12499]

55. Malchiodi L, Ghensi P, Cucchi A, Pieroni S, Bertossi D. Peri-implant conditions around sintered porous-surfaced (SPS) implants. A 36-month prospective cohort study. Clin Oral Implants Res. 2015 Feb;26(2):212-9. [Medline: 24383851] [doi: $10.1111 / \mathrm{clr} .12311]$

56. Hita-Iglesias C, Sánchez-Sánchez FJ, Montero J, Galindo-Moreno P, Mesa F, Martínez-Lara I, Sánchez-Fernández E. Immediate implants placed in fresh sockets associated with periapical pathology: a split-mouth design and survival evaluation after 1-year follow-up. Clin Implant Dent Relat Res. 2015 Dec 16. [Medline: 26676203] [doi: $10.1111 /$ cid.12387]

57. Daubert DM, Weinstein BF, Bordin S, Leroux BG, Flemming TF. Prevalence and predictive factors for peri-implant disease and implant failure: a cross-sectional analysis. J Periodontol. 2015 Mar;86(3):337-47. [Medline: 25415249] [doi: 10.1902/jop.2014.140438]

58. Gherlone EF, Capparé P, Tecco S, Polizzi E, Pantaleo G, Gastaldi G, Grusovin MG. A prospective longitudinal study on implant prosthetic rehabilitation in controlled HIV-positive patients with 1-year follow-up: the role of CD4+ level, smoking habits, and oral hygiene. Clin Implant Dent Relat Res. 2015 Aug 4. [Medline: 26238779] [doi: 10.1111/cid.12370]

59. Konermann A, Götz W, Le M, Dirk C, Lossdörfer S, Heinemann F. Histopathological verification of osteoimmunological mediators in peri-implantitis and correlation to bone loss and implant functional period. J Oral Implantol. 2016 Feb;42(1):61-8. [Medline: 24945460] [doi: 10.1563/aaid-joi-D-13-00355]

60. Tomasi C, Derks J. Clinical research of peri-implant diseases--quality of reporting, case definitions and methods to study incidence, prevalence and risk factors of peri-implant diseases. J Clin Periodontol. 2012 Feb;39(Suppl 12):207-23. [Medline: 22533958] [doi: 10.1111/j.1600-051X.2011.01831.x]

61. Heitz-Mayfield LJ, Huynh-Ba G. History of treated periodontitis and smoking as risks for implant therapy. Int J Oral Maxillofac Implants. 2009;24(Suppl):39-68. [Medline: 19885434] 
62. Lindhe J, Meyle J. Group D of European Workshop on Periodontology. Peri-implant diseases: Consensus Report of the Sixth European Workshop on Periodontology. J Clin Periodontol. 2008 Sep;35(8 Suppl):282-5. [Medline: 18724855] [doi: 10.1111/j.1600-051X.2008.01283.x]

63. Albrektsson T, Buser D, Chen ST, Cochran D, DeBruyn H, Jemt T, Koka S, Nevins M, Sennerby L, Simion M, Taylor TD, Wennerberg A. Statements from the Estepona consensus meeting on peri-implantitis, February 2-4, 2012. Clin Implant Dent Relat Res. 2012 Dec;14(6):781-2. [Medline: 23205721] [doi: 10.1111/cid.12017]

64. Canullo L, Schlee M, Wagner W, Covani U; Montegrotto Group for the Study of Peri-implant Disease. International Brainstorming Meeting on Etiologic and Risk Factors of Peri-implantitis, Montegrotto (Padua, Italy), August 2014. Int J Oral Maxillofac Implants. 2015 Sep-Oct;30(5):1093-104. [Medline: 26394346] [doi: 10.11607/jomi.4386]

65. Barone A, Alfonsi F, Derchi G, Tonelli P, Toti P, Marchionni S, Covani U. The effect of insertion torque on the clinical outcome of single implants: a randomized clinical Trial. Clin Implant Dent Relat Res. 2015 Jun 5. [Medline: 26043651] [doi: $10.1111 /$ cid.12337]

66. Hansson S, Werke M. The implant thread as a retention element in cortical bone: the effect of thread size and thread profile: a finite element study. J Biomech. 2003 Sep;36(9):1247-58. [Medline: 12893033] [doi: 10.1016/S0021-9290(03)00164-7]

67. Monje A, Galindo-Moreno P, Tözüm TF, Suárez-López Del Amo F, Wang HL. Into the paradigm of local factors as contributors for peri-implant disease: short communication. Int J Oral Maxillofac Implants. 2016 Mar-Apr;31(2):288-92. [Medline: 27004275] [doi: 10.11607/jomi.4265]

68. Spray JR, Black CG, Morris HF, Ochi S. The influence of bone thickness on facial marginal bone response: stage 1 placement through stage 2 uncovering. Ann Periodontol. 2000 Dec;5(1):119-28. [Medline: 11885170] [doi: 10.1902/annals.2000.5.1.119]

69. Chen ST, Darby IB, Reynolds EC. A prospective clinical study of non-submerged immediate implants: clinical outcomes and esthetic results. Clin Oral Implants Res. 2007 Oct;18(5):552-62. [Medline: 17608739] [doi: 10.1111/j.1600-0501.2007.01388.x]

70. Fu JH, Hsu YT, Wang HL. Identifying occlusal overload and how to deal with it to avoid marginal bone loss around implants. Eur J Oral Implantol. 2012;5 Suppl:S91-103. [Medline: 22834398]

71. Sgolastra F, Petrucci A, Severino M, Gatto R, Monaco A. Smoking and the risk of peri-implantitis. A systematic review and meta-analysis. Clin Oral Implants Res. 2015 Apr;26(4):e62-7. [Medline: 24438442] [doi: 10.1111/clr.12333]

72. Chrcanovic BR, Albrektsson T, Wennerberg A. Smoking and dental implants: a systematic review and meta-analysis. J Dent. 2015 May;43(5):487-98. [Medline: 25778741] [doi: 10.1016/j.jdent.2015.03.003]

73. Shrier I, Boivin JF, Steele RJ, Platt RW, Furlan A, Kakuma R, Brophy J, Rossignol M. Should meta-analyses of interventions include observational studies in addition to randomized controlled trials? A critical examination of underlying principles. Am J Epidemiol. 2007 Nov 15;166(10):1203-9. [Medline: 17712019] [doi: 10.1093/aje/kwm189]

\section{To cite this article:}

Stacchi C, Berton F, Perinetti G, Frassetto A, Lombardi T, Khoury A, Andolsek F, Di Lenarda R.

Risk Factors for Peri-Implantitis: Effect of History of Periodontal Disease and Smoking Habits. A Systematic Review and Meta-Analysis

J Oral Maxillofac Res 2016;7(3):e3

URL: http://www.ejomr.org/JOMR/archives/2016/3/e3/v7n3e3.pdf

doi: $10.5037 /$ jomr.2016.7303

Copyright (C) Stacchi C, Berton F, Perinetti G, Frassetto A, Lombardi T, Khoury A, Andolsek F, Di Lenarda R. Published in the JOURNAL OF ORAL \& MAXILLOFACIAL RESEARCH (http://www.ejomr.org), 9 September 2016.

This is an open-access article, first published in the JOURNAL OF ORAL \& MAXILLOFACIAL RESEARCH, distributed under the terms of the Creative Commons Attribution-Noncommercial-No Derivative Works 3.0 Unported License, which permits unrestricted non-commercial use, distribution, and reproduction in any medium, provided the original work and is properly cited. The copyright, license information and link to the original publication on (http://www.ejomr.org) must be included. 